\title{
Propofol inhibits the growth and survival of gastric cancer cells in vitro through the upregulation of ING3
}

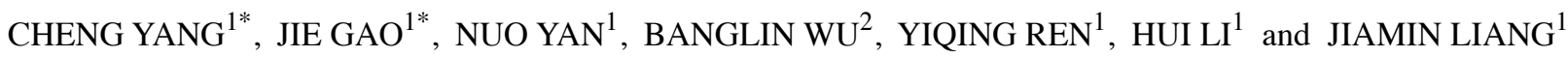 \\ ${ }^{1}$ Department of Anesthesiology, Affiliated Hospital of Logistics University of PAP, Tianjin 300162; \\ ${ }^{2}$ Department of Anesthesiology, The Central Hospital of Enshi Autonomous Prefecture, Enshi, Hubei 445000, P.R. China
}

Received May 4, 2016; Accepted June 17, 2016

DOI: $10.3892 /$ or.2016.5218

\begin{abstract}
Propofol is one of the most extensively used intravenous anesthetic agents and it can influence the biological behavior of gastric cancer. However, the underlying mechanism is poorly understood. In the present study, we found that propofol significantly inhibited cell proliferation, invasion and migration, and also promoted apoptosis in gastric carcinoma cell lines SGC-7901 and MGC-803, as detected using MTT, colony formation and flow cytometry assays, respectively. Moreover, propofol (10 and $20 \mu \mathrm{M})$ markedly upregulated the expression of inhibitor of growth 3 (ING3), which was lower in SGC-7901 and MGC-803 cells compared with that noted in normal human gastric epithelial cell lines GES-1 and HFE145. Furthermore, we transfected SGC-7901 and MGC-803 cells with ING3 overexpression vectors or ING3 small interference RNA (siING3), respectively, to assess the role of ING3 in propofol-induced antitumor activity. The siING3 transfection reversed the effects of propofol on the biological behavior of gastric cancer cells, while transfection of ING3 promoted the effects of propofol. In conclusion, our results indicate that propofol exerts an inhibitory effect on the growth and survival of gastric cancer cells by interfering with ING3 degradation.
\end{abstract}

\section{Introduction}

Gastric cancer is the fourth most common cancer with high morbidity and mortality worldwide $(1,2)$. Approximately $70 \%$ of new gastric cancer cases and gastric cancer-related deaths occur in developing countries (3). Surgery is regarded as the first line treatment for solid tumors, such as gastric cancer.

Correspondence to: Dr Cheng Yang, Department of Anesthesiology, Affiliated Hospital of Logistics University of PAP, 220 Chenglin Road, Tianjin 300162, P.R. China

E-mail: chengyangtj@163.com

${ }^{*}$ Contributed equally

Key words: propofol, gastric cancer, inhibitor of growth 3
Globally, there are 234 million individuals undergoing surgery with anesthesia each year (4). Recently, general anesthesia was reported to be associated with the risk of malignant tumor recurrence (5). Therefore, the role of anesthesia is vital for cancer development.

The inhibitor of growth family member 3 (ING3) is a member of the ING tumor-suppressor family (6), which is mapped to 7q31.3 and consists of 12 exons. ING3 was reported to play a significant role in modulating transcription, cell cycle and apoptosis $(7,8)$. It could link the function of p53 to histone acetylation (9). ING3 was also reported to be reduced in human head and neck squamous cell carcinomas (10). ING3 also plays pivotal roles in the progression of human hepatocellular carcinoma (11) and colorectal adenoma, and may be a potential target for cancer diagnosis and therapy (12). Although its expression has been reported in various types of cancer, the role of ING3 in gastric cancer has not been investigated.

Propofol (2,6-diisopropylphenol) is one of the most widely used intravenous anesthetic agents during cancer resection surgery. In addition to its anesthetic properties, propofol also exhibits antitumor effects $(13,14)$, which can inhibit proliferation and invasiveness, and induce apoptosis of cancer cells. Numerous studies have reported that propofol has an inhibitory effect on cancer cells. For example, propofol inhibited lung cancer cell proliferation and induced its apoptosis (15). It has also shown an inhibitory effect on the invasion and metastasis of cancer cells such as lung (15), ovarian (16) and prostate cancer (17), and osteosarcoma (18). There is no doubt that the antitumor effect of propofol is extremely advantageous for the surgical treatment of tumor patients. Recent studies have demonstrated that propofol inhibited the growth of gastric cancer cells (19). However, the molecular mechanisms by which it acts remain unclear. In the present study, we used gastric cancer cell lines MGC-803 and SGC-7901 to explore the effects and the mechanism of action of propofol on gastric cancer cells.

\section{Materials and methods}

Cell culture and treatment. Human gastric cancer cell lines (MGC-803 and SGC-7901) and normal human gastric epithelial cell lines (GES-1 and HFE145) were obtained from the Type Culture Collection of the Chinese Academy of Sciences 
(Shanghai, China). The cells were grown in Dulbecco's $\mu$ odified Eagle's medium (DMEM; Gibco, Rockville, MD, USA) containing $10 \%$ fetal bovine serum (FBS; Invitrogen, Carlsbad, CA, USA), $100 \mathrm{U} / \mathrm{ml}$ penicillin and $100 \mu \mathrm{g} / \mathrm{ml} \mathrm{strep-}$ tomycin (Gibco) and were maintained in a humidified $5 \% \mathrm{CO}_{2}$ atmosphere at $37^{\circ} \mathrm{C}$.

Transfection. MGC-803 and SGC-7901 cells were respectively transfected with ING3 plasmid (pcDNA3-ING3) or ING3 siRNA plasmid (pcDNA3-siING3) or negative control plasmid (pcDNA3-NC) using Lipofectamine 2000 reagent (Invitrogen) according to the operating protocols. The ING3 siRNA sequences (Ambion, Austin, TX, USA) were: 5'-GCUGAUAA UGCUGGAAUUAUU-3' (sense) and 5'-UAAUUCCAGCAU UAUCAGCUU-3' (antisense) (20). Twenty-four hours later, stable transfected cells were selected with G418 (Sigma, St. Louis, MO, USA) and cultured for further study. Transfection efficiency was monitored by qPCR and western blotting.

Propofol treatment. To determine the effect of propofol on gastric cancer, MGC-803 and SGC-7901 cells were exposed to $0,5,10$ or $20 \mu \mathrm{M}$ propofol (Sigma) for $72 \mathrm{~h}$ before collection for further analysis. Propofol was diluted with dimethyl sulfoxide (DMSO; Sigma).

Cell viability. Cell viability of the gastric cancer cells was measured by 3-(4,5-dimethylthiazol-2-yl)-2,5-diphenyltetrazolium bromide (MTT; Sigma) assay. Briefly, the cells were seeded on 96-well plates $\left(5 \times 10^{3}\right.$ cells/well) and treated with propofol for $72 \mathrm{~h}$. Then, $20 \mu \mathrm{l}$ of MTT ( $5 \mathrm{mg} / \mathrm{ml}$ ) was added into each well and incubated for another $4 \mathrm{~h}$. The supernatant was aspirated. DMSO (200 $\mu \mathrm{l})$ was added to each well to dissolve MTT formazan. The absorbance at $490 \mathrm{~nm}$ was measured using a microplate reader (BMG Labtech, Inc., Durham, NC, USA). Viability $=$ OD treatment $/$ OD control $\times 100 \%$. The experiments were repeated three times independently.

Migration and invasion assays. The invasion assay was performed using a 24-well invasion chamber system coated with $50 \mu \mathrm{l}$ diluted Matrigel (BD Biosciences, Bedford, MA, USA) according to the manufacturer's instructions. Cells were seeded in the upper chamber $\left(1 \times 10^{5}\right.$ cells/well $)$ in serum-free media. The lower chamber was filled with media containing $10 \%$ FBS. After incubation for $24 \mathrm{~h}$, the migrating cell number was determined by counting five random x100 fields on each membrane under a microscope (Zeiss, Oberkochen, Germany), and the mean values from three independent experiments were used. A similar procedure was used for the invasion assay. The only difference was that the upper chamber was not coated with Matrigel.

Flow cytometry. Cell apoptosis was detected with Annexin V-FITC and propidium iodide (PI) staining followed by flow cytometric (Beckman Coulter Epics XL; Beckman Coulter, Krefeld, Germany) analysis. Briefly, the cells were harvested and seeded into 96-well plates and treated with propofol. A total of $3 \times 10^{5}$ cells were stained with Annexin V-FITC/PI using the Annexin V-FITC/PI kit (BD Pharmingen, San Diego, CA, USA).
Quantitative real-time PCR ( $q P C R)$. Total RNA was extracted from gastric cancer MGC-803 and SGC-7901 and gastric epithelial cells GES-1 and HFE145 using TRIzol reagent (Invitrogen). Reverse transcription was carried out using the M-MLV Reverse Transcriptase System (Clontech, Palo Alto, CA, USA), and qPCR was performed using SYBR Premix Ex Taq (Takara, Dalian, China). GAPDH was used for normalization. The primer sequences were as follows (12): ING3 forward, 5'-CTTCACGGAAATGCG-3' and reverse, 5'-CTCTTCCCT CCACTCA-3'; GAPDH forward, 5'-CTCAGACACCATGGG GAAGGTGA-3' and reverse, 5'-ATGATCTT2GAGGCTGTT GTCATA-3'. The $2^{-\Delta \Delta C t}$ method was used to quantify the relative gene expression. The relative expression of ING3 was normalized to the expression of GAPDH.

Western blot analysis. Total protein was extracted from cells and separated on $12 \%$ SDS-polyacrylamide gel. After electrophoresis, the proteins were transferred to nitrocellulose membranes (Amersham, Little Chalfont, UK), which were then blocked in $5 \%$ fat-free milk for $1 \mathrm{~h}$ at $37^{\circ} \mathrm{C}$. Subsequently, the membranes were probed with the primary antibody for ING3 $(1: 1,000)$ or GAPDH $(1: 1,000)$ (both from Abcam, Cambridge, UK) at $4^{\circ} \mathrm{C}$ overnight. Then, they were incubated with HRP-conjugated anti-rabbit IgG secondary antibody (1:4,000; Abcam) for $2 \mathrm{~h}$, and then washed with Tris-buffered saline containing Tween-20 (TBST). The proteins were finally visualized using chemiluminescent ECL reagent (Santa Cruz Biotechnology, Santa Cruz, CA, USA) with ImageJ software (Media Cybernetics, Inc., Rockville, MD, USA).

Statistical analysis. All values are reported as mean \pm SD. Statistical analysis was performed using SPSS 20.0 (SPSS, Inc., Chicago, IL, USA) by either two-sided Student's unpaired $\mathrm{t}$-test or ANOVA test with post $\mathrm{t}$-test. Statistical significance was accepted at P-values $<0.05$.

\section{Results}

Propofol inhibits gastric cancer cell growth and induces cell apoptosis. To elucidate the role of propofol in gastric cancer, we first investigated the effects of propofol on cell proliferation, invasion, migration and apoptosis. MGC-803 and SGC-7901 cells treated with propofol (10 and $20 \mu \mathrm{M})$ showed markedly decreased cell proliferation (Fig. 1A and B), invasion (Fig. 1C and D) and migration (Fig. 1E and F), and increased apoptosis (Fig. $1 \mathrm{G}$ and $\mathrm{H}$ ) in comparison with the untreated cells $(\mathrm{P}<0.05)$.

ING3 is expressed at low levels in human gastric cancer cells. To investigate whether ING3 plays a role in gastric cancer, we used two gastric cancer cell lines (MGC-803 and SGC-7901) and two normal gastric epithelial cell lines (CES-1 and HFE145) to analyze ING3 expression. The results showed that both the mRNA expression and protein level of ING3 were weak in the MGC-803 and SGC-7901 cells compared to these levels in the CES-1 and HFE145 cells ( $\mathrm{P}<0.05$; Fig. 2A and B), as demonstrated by qPCR and western blot analysis, respectively. This indicates that ING3 may play a crucial role in the development of gastric cancer. To determine the critical role of ING3 in gastric cancer, we generated MGC-803 and 
A

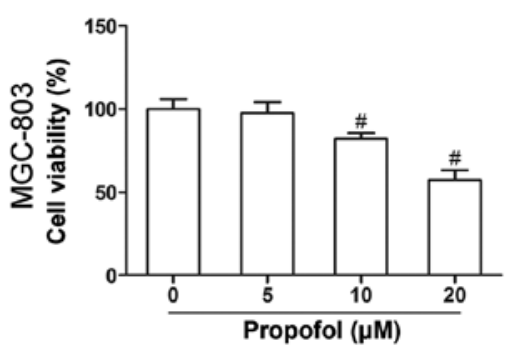

B

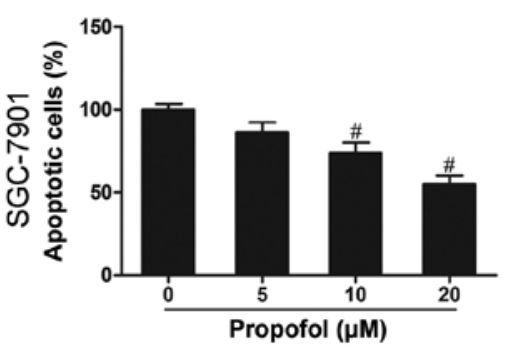

C

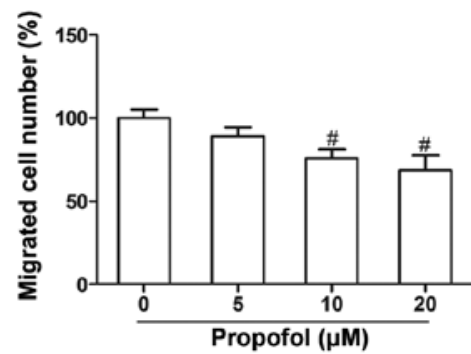

D

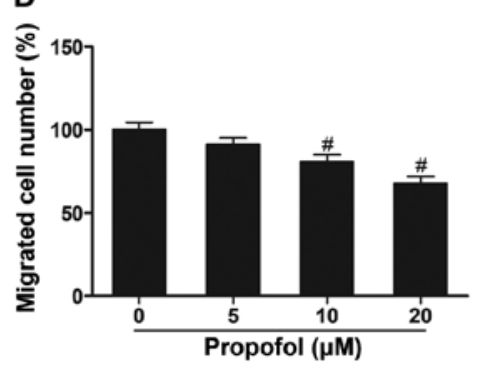

E

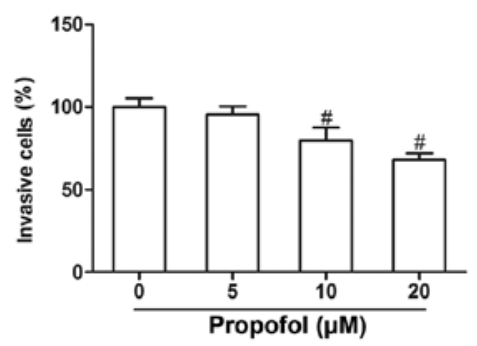

F

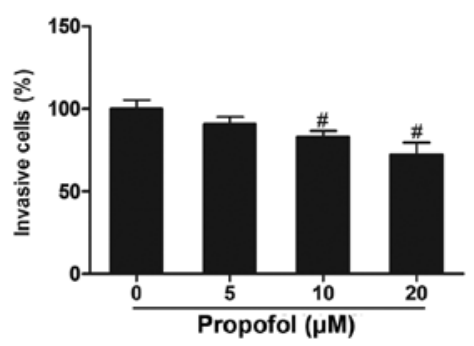

G

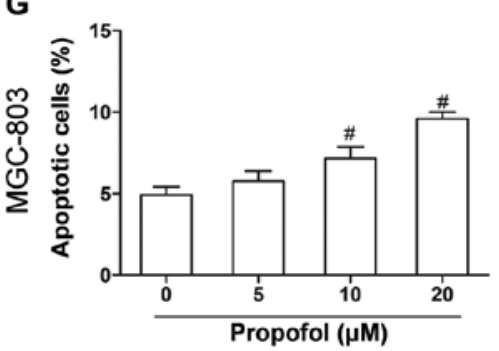

H

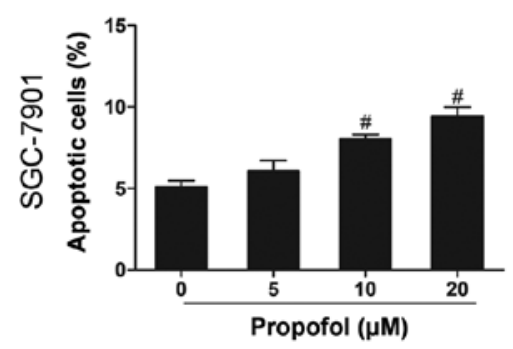

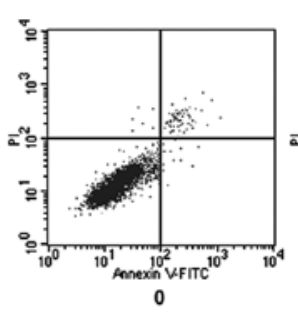
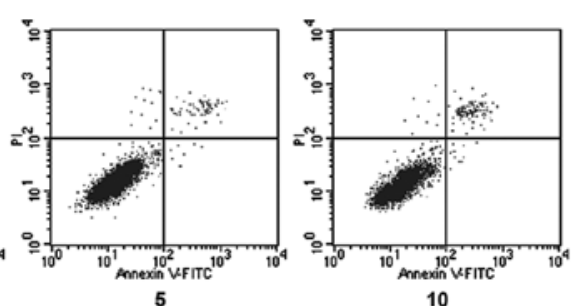

10

Propofol $(\mu \mathrm{M})$
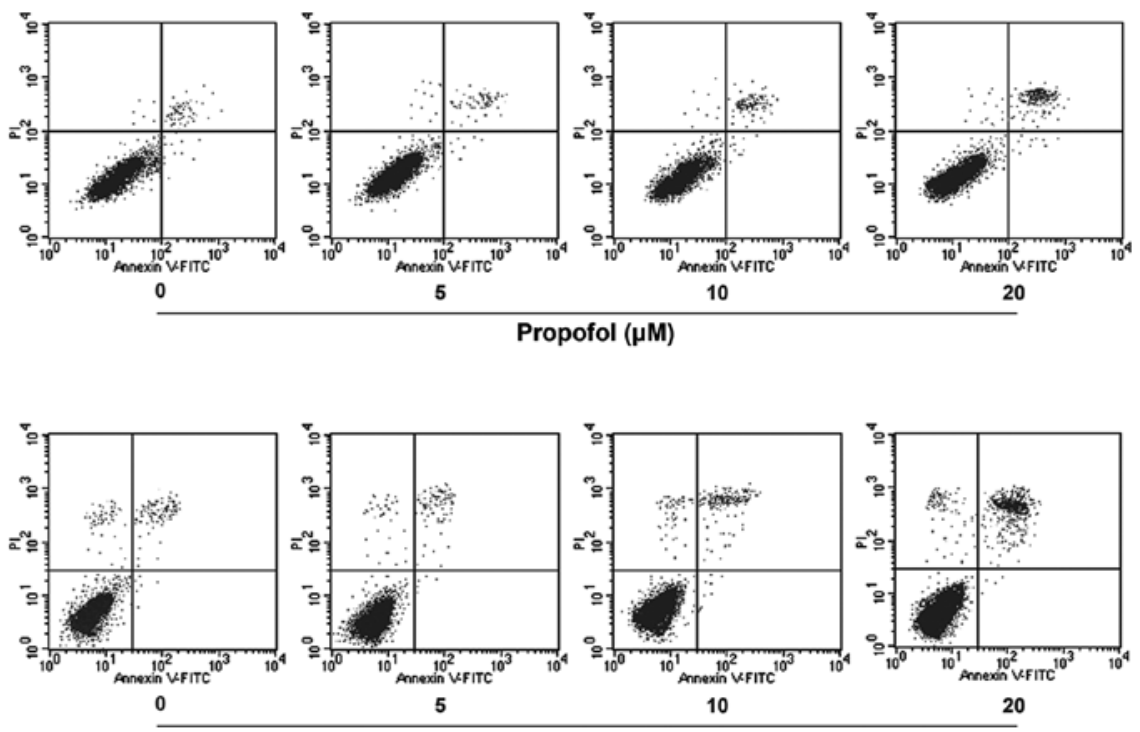

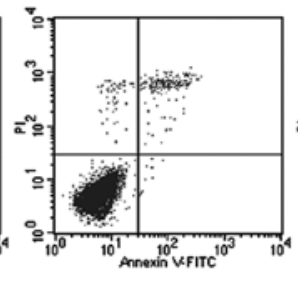

10

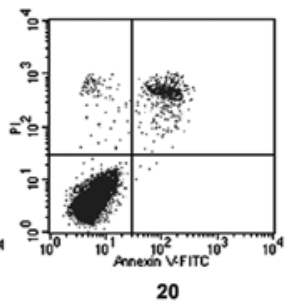

20

Figure 1. Propofol suppresses gastric cancer cell growth and induces cell apoptosis. Propofol (10 and $20 \mu \mathrm{M})$ treatment inhibited (A and B) cell proliferation, $(\mathrm{C}$ and $\mathrm{D})$ migration and $(\mathrm{E}$ and $\mathrm{F})$ invasion, and promoted $(\mathrm{G}$ and $\mathrm{H})$ apoptosis in the MGC-803 and SGC-7901 cells. N=3, ${ }^{\#} \mathrm{P}<0.05 \mathrm{vs}$. propofol $(0 \mu \mathrm{M})$.

SGC-7901 cells that stably expressed ING3 or siING3, in which the expression was significantly upregulated or downregulated (Fig. 2C-F) $(\mathrm{P}<0.05)$.

ING3 suppresses the survival and induces apoptosis of gastric cancer cells. We then evaluated the effect of ING3 on the growth of gastric cancer cells. The results revealed that ING3 also significantly inhibited cell proliferation (Fig. 3A and B), invasion (Fig. 3C and D) and migration (Fig. 3E and F), and increased apoptosis (Fig. 3G and $\mathrm{H}$ ) compared with the negative control and control groups $(\mathrm{P}<0.05)$. However, siING3 markedly promoted cell proliferation (Fig. 3A and B), invasion (Fig. 3C and D) and migration (Fig. 3E and F), and decreased apoptosis (Fig. 3G and H) of the MGC-803 and SGC-7901 cells $(\mathrm{P}<0.05)$.
Propofol increases the expression of ING3 in human gastric cancer cells. To study the correlation of propofol and ING3 in gastric cancer, we detected ING3 expression in propofol-treated MGC-803 and SGC-7901 cells. As shown in Fig. 4A-D, propofol-untreated cells induced low levels of expression of ING3, while propofol-treated (10 and $20 \mu \mathrm{M})$ cells showed strong ING3 expression, demonstrating that the ING3 level was increased by propofol.

ING3 promotes the effect of propofol on cell growth. To further elucidate whether ING3 was responsible for the effect of propofol on gastric cancer, we treated cells that were transfected with ING3 or siING3 with propofol $(10 \mu \mathrm{M})$. After administration of propofol $(10 \mu \mathrm{M})$, ING3-transfected cells showed a significantly decreased cell proliferation (Fig. 4E and F), 
A

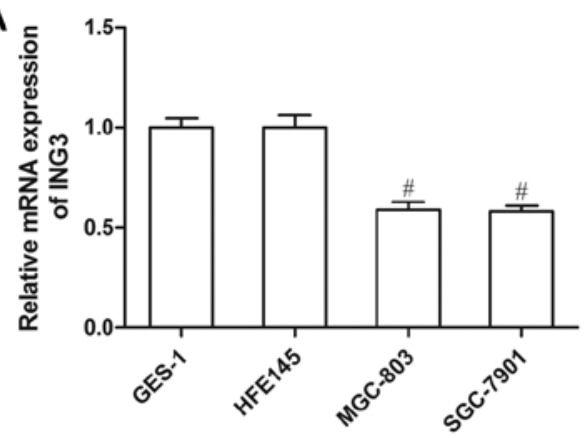

C

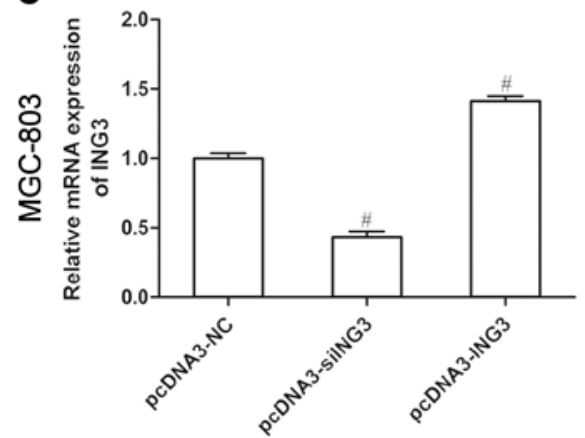

E

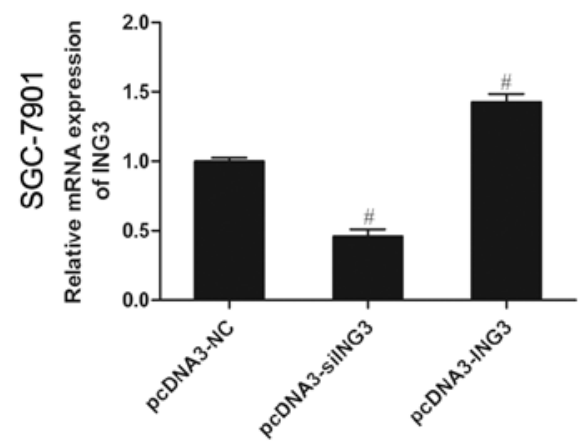

B

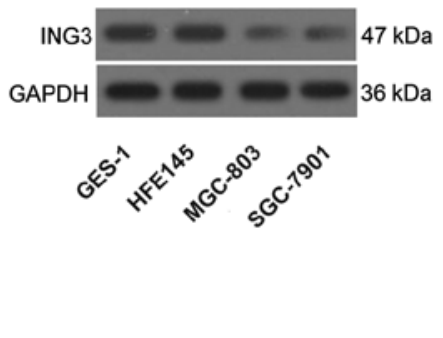

D

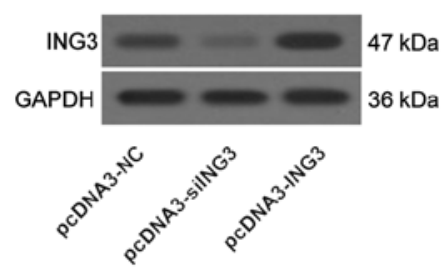

$\mathbf{F}$

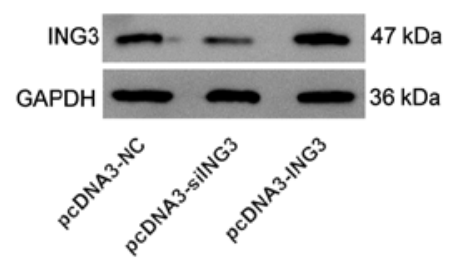

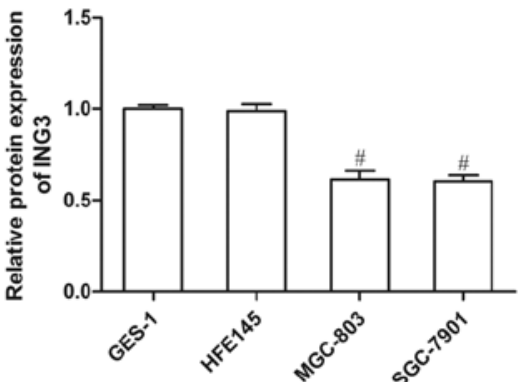
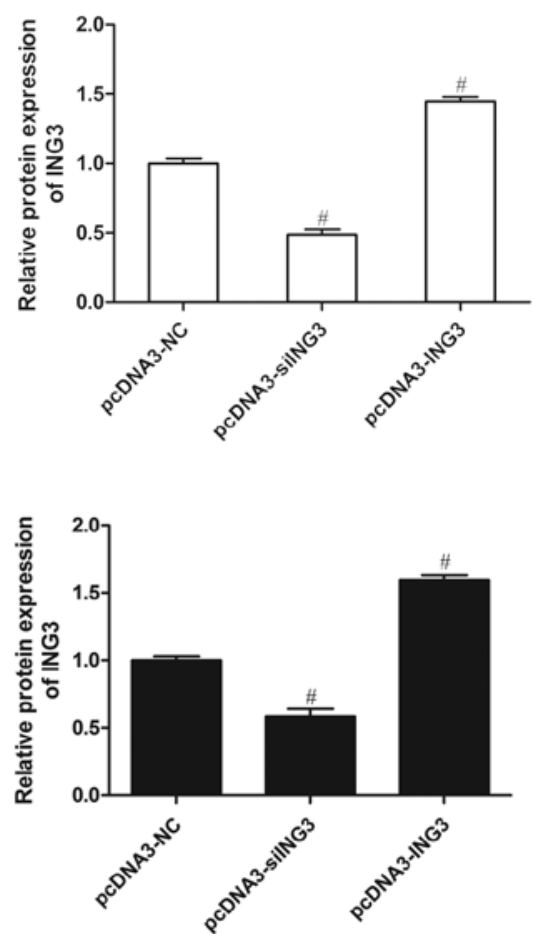

Figure 2. ING3 is expressed at low levels in human gastric cancer cells. (A) mRNA expression and (B) protein level of ING3 were lower in gastric cancer cell lines (MGC-803 and SGC-7901) than in normal gastric epithelial cell lines (CES-1 and HFE145) as analyzed by qPCR and western blot analysis. N=3, ${ }^{\#} \mathrm{P}<0.05$ vs. CES-1 and HFE145 cells. ING3 expression was increased in the MGC-803 and SGC-7901 cells transfected with ING3, but decreased in cells transfected with siING3 according to (C and E) qPCR and (D and F) western blot analysis. $\mathrm{N}=3,{ }^{\#} \mathrm{P}<0.05$ vs. pcDNA3-NC.

invasion (Fig. 4G and $\mathrm{H}$ ) and migration (Fig. 4I and J), and an increased apoptosis (Fig. 4K and L) in the MGC-803 and SGC-7901 cells. In contrast, small interfering RNA (siRNA)mediated knockdown of ING3 in the MGC-803 and SGC-7901 cells, even those treated with propofol $(10 \mu \mathrm{M})$, exhibited significantly increased cell proliferation (Fig. 4E and F), invasion (Fig. 4G and $\mathrm{H}$ ) and migration (Fig. 4I and $\mathrm{J}$ ), and reduced apoptosis (Fig. $4 \mathrm{~K}$ and L) compared with the untreated MGC-803 and SGC-7901 cells. In other words, siING3 reversed the effects of propofol, while ING3 promoted the effects of propofol on cell growth.

\section{Discussion}

Gastric cancer is the second leading cause of cancer-related death worldwide (21), with a low 5-year survival rate (2). Mortality usually results from recurrence or metastases. Surgical removal of the primary tumor is of utmost importance for treatment (22). The increasing incidence of cancer means that anesthesia is important, not only during the perioperative period, but also during chronic cancer pain management (23). It was reported that anesthetic agents influence the pathophysiology of metastasis in the postoperative period (23). An appropriate anesthetic strategy could improve the longterm survival of patients. Anesthetic agents and anesthesia techniques play pivotal roles in the prevention of tumor metastasis and recurrence.

In the present study, we found that propofol suppressed the growth of both MGC-803 and SGC-7901 cells. Lower ING3 expression was found in the MGC-803 and SGC-7901 cells than that noted in the CES-1 and HFE145 cells. Propofol also increased ING3 expression, affecting the biological behavior of gastric cancer cells. We further found that ING3 was associated with the inhibitory effect of propofol on MGC-803 and SGC-7901 cells. Together, our studies provide evidence that propofol is an important inhibitor of gastric cancer progression in vitro by upregulating ING3, and suggest that ING3 is a promising therapeutic target for gastric cancer. 
A

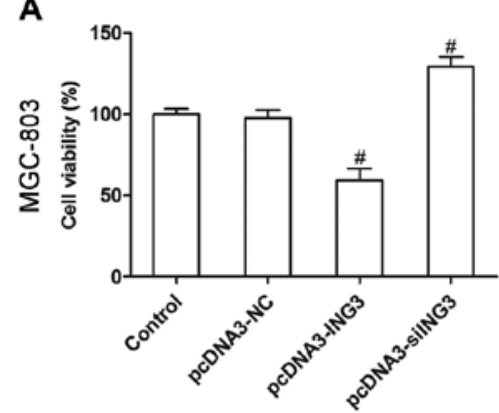

B

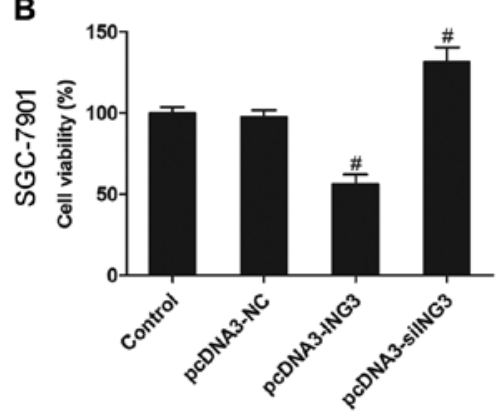

C

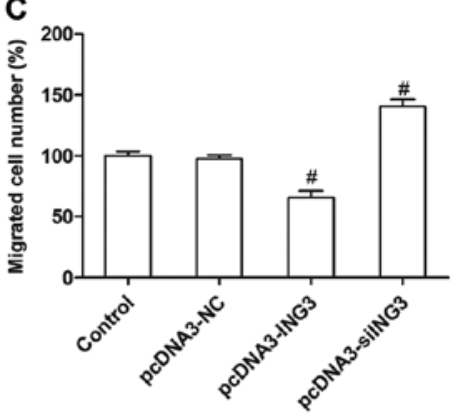

D

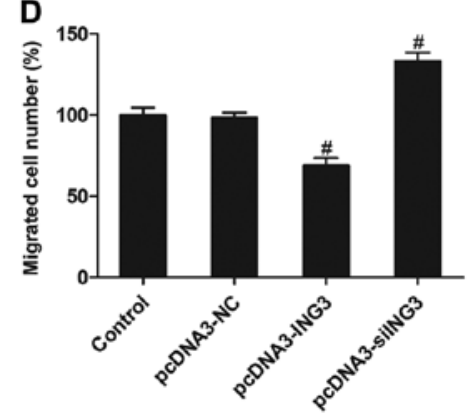

E

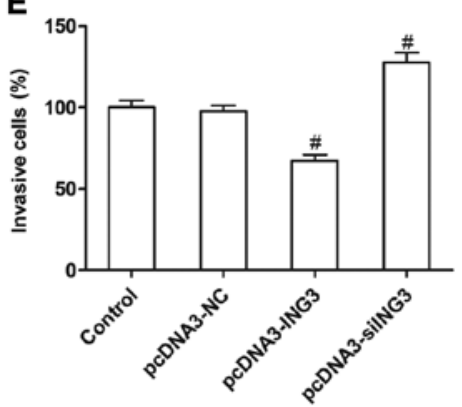

$\mathbf{F}$

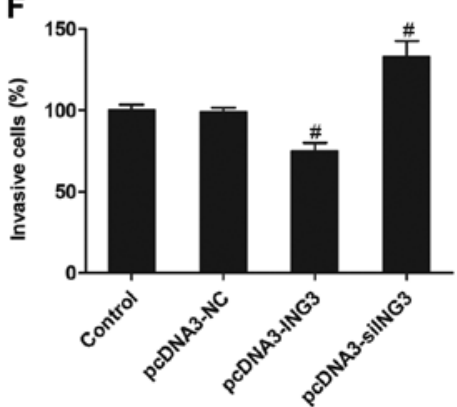

G
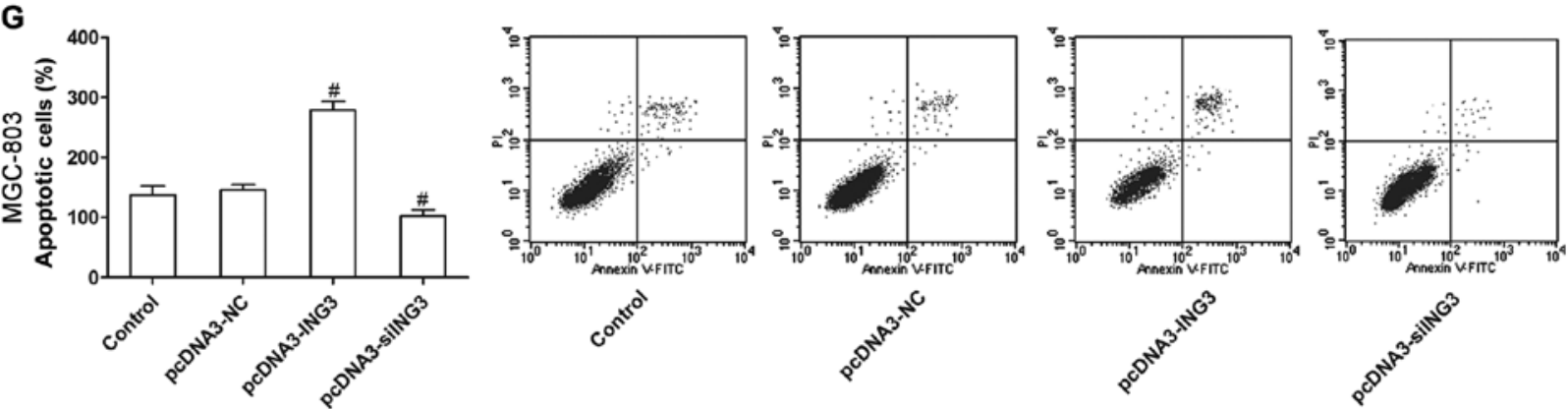

H
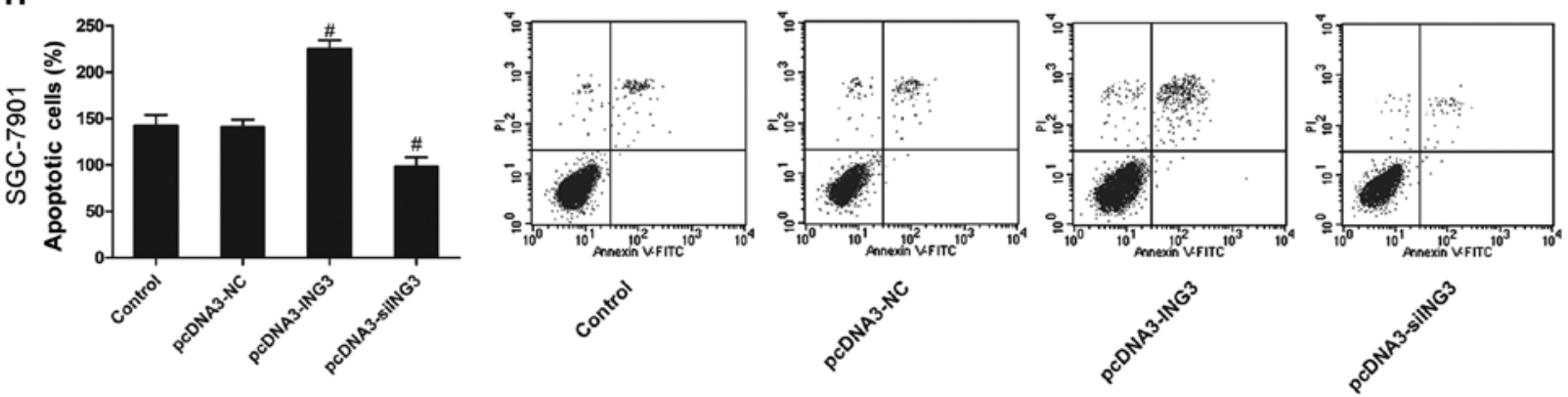

Figure 3. ING3 suppresses gastric cancer cell growth and induces cell apoptosis. ING3 transfection reduced (A and B) cell proliferation, (C and D) migration and $(\mathrm{E}$ and $\mathrm{F})$ invasion, and promoted $(\mathrm{G}$ and $\mathrm{H})$ apoptosis in the MGC-803 and SGC-7901 cells. $\mathrm{N}=3,{ }^{\#} \mathrm{P}<0.05$ vs. control.

Propofol, a commonly used intravenous anesthetic, has been reported to exert many non-anesthetic effects (24). Increasing evidence suggests that propofol suppresses the proliferation $(25,26)$, adhesion $(27,28)$ and invasion $(29,30)$ of cancer cells, and induces their apoptosis $(31,32)$. Therefore, it may be beneficial to use propofol in cancer surgery, for reasons other than its multiple anesthetic advantages. In the present study, we verified that propofol was effective at inhibiting the growth of gastric cancer cells, which was consistent with previous studies (19). Moreover, propofol enhanced the expression of ING3.

ING3, a candidate tumor-suppressor of the ING family, plays an unquestionable role in modulating transcription, cell cycle and apoptotic induction (33). Its downregulation may contribute to tumorigenesis. Numerous studies have suggested that ING3 is a functional tumor-suppressor gene in human cancers (10-12). In the present study, ING3 expression in gastric cancer cells was found to be lower than its expression in normal gastric epithelial cells. These findings indicate that a downregulated ING3 level may be involved in gastric carcinogenesis. ING3 transfection markedly promoted the inhibitory effects of propofol on gastric cells, whereas siING3 transfection reversed the effects of propofol, suggesting that propofol plays a role in modulating gastric cancer cells by targeting ING3.

In conclusion, the present study provides new insights into the effects of propofol on the behavior of gastric cancer cells 


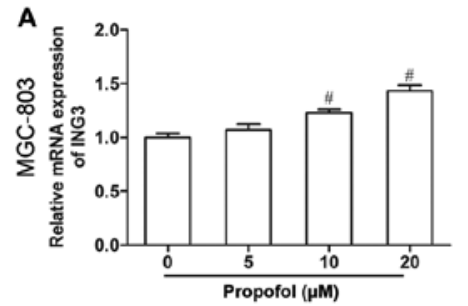

B

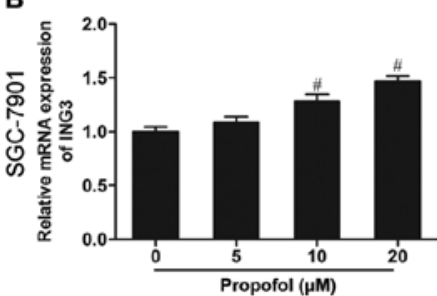

E

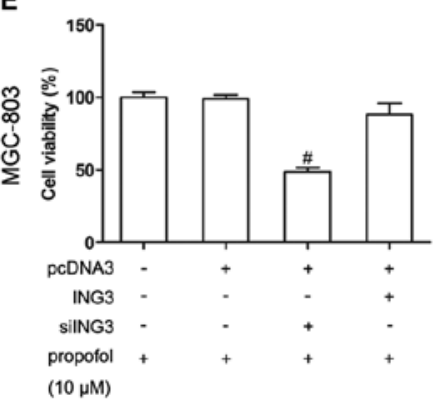

$\mathbf{F}$
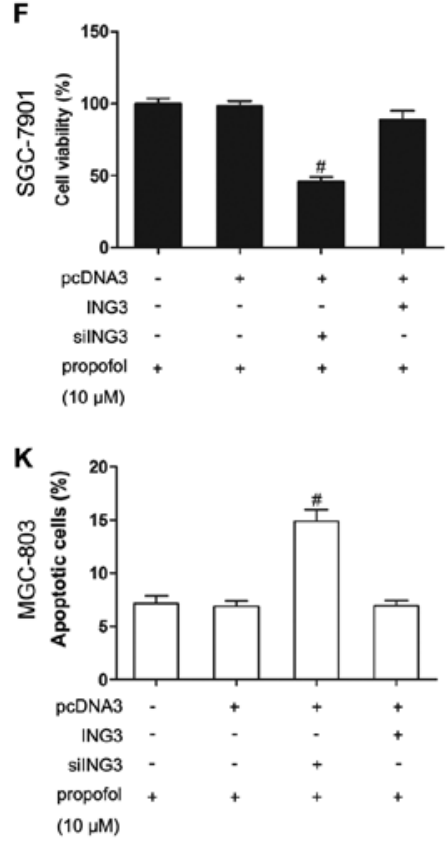

$\mathbf{L}$

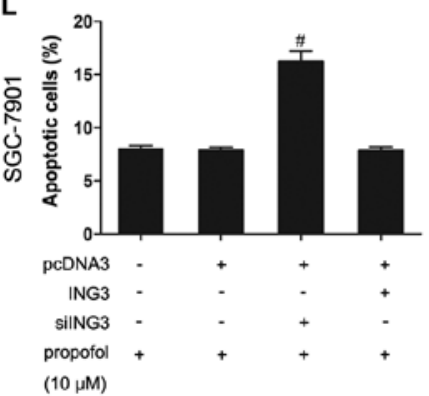

C

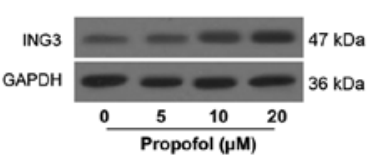

D

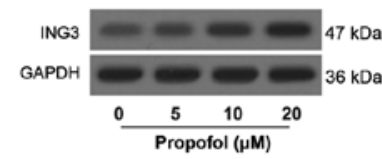

G

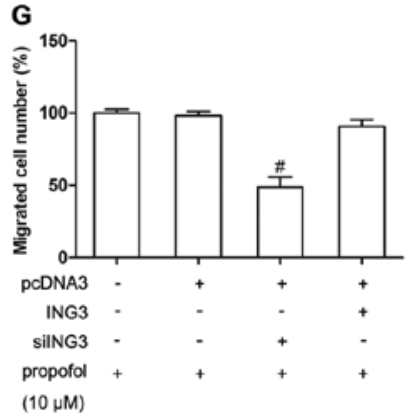

H

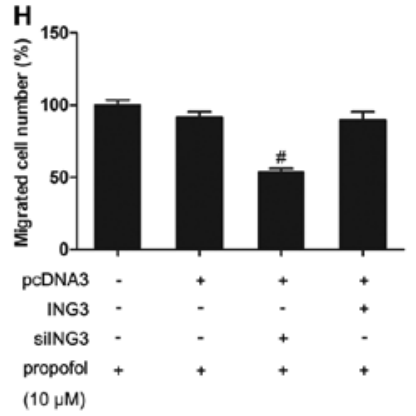

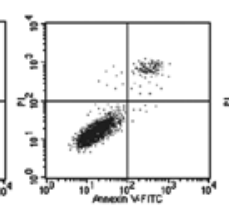
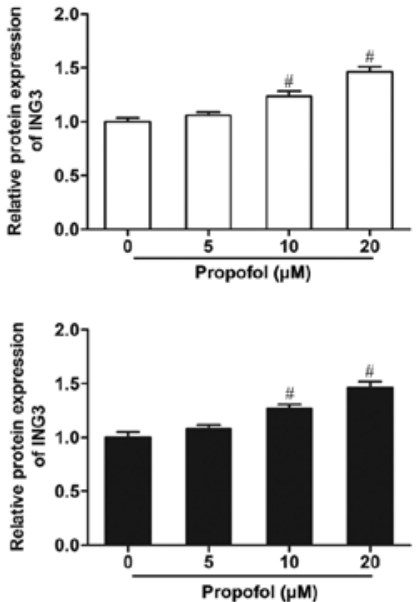

I
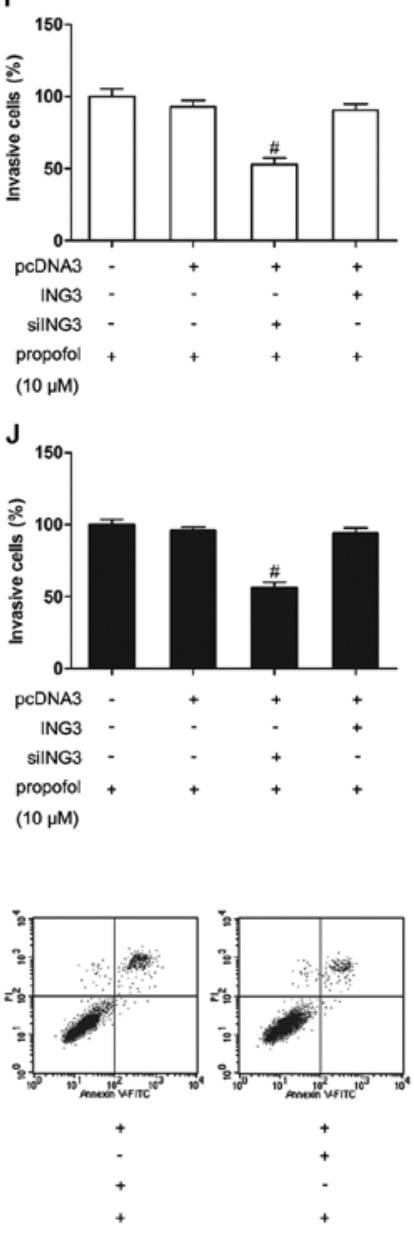

$(10 \mu \mathrm{M})$
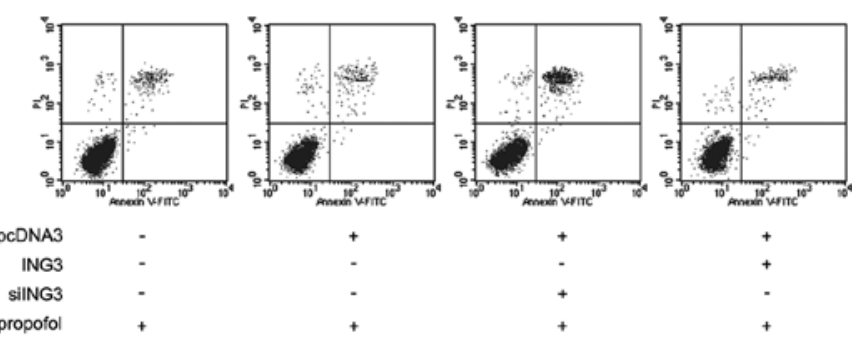

$(10 \mu \mathrm{M})$

Figure 4. Propofol restrains human gastric cell growth by upregulating ING3. Propofol (10 and $20 \mu \mathrm{M})$ upregulated the (A and B) mRNA expression and $\left(\mathrm{C}\right.$ and D) protein -level of ING3 in MGC-803 and SGC-7901 cells. N=3, ${ }^{\#} \mathrm{P}<0.05$ vs. propofol $(0 \mu \mathrm{M})$. Transfection of ING3 promoted the effects of propofol $(10 \mu \mathrm{M})$, inhibiting (E and F) cell proliferation, (G and H) migration and (I and J) invasion, and promoting (K and L) apoptosis of MGC-803 and SGC-7901 cells; transfection of siING3 reversed the effects of propofol $(10 \mu \mathrm{M})$. $\mathrm{N}=3,{ }^{\#} \mathrm{P}<0.05$ vs. cells only treated with propofol $(10 \mu \mathrm{M})$. 
and the mechanisms involved. Propofol may have antitumor potential in gastric cancer, partly due to the upregulation of ING3.

\section{Acknowledgements}

The present study was supported by grants from the Science and Technology of Tianjin Health Bureau (no. 2015kz124).

\section{References}

1. Yoon $\mathrm{H}$ and Kim N: Diagnosis and management of high risk group for gastric cancer. Gut Liver 9: 5-17, 2015.

2. Zhang S, Chen P, Huang Z, Hu X, Chen M, Hu S, Hu Y and Cai T: Sirt7 promotes gastric cancer growth and inhibits apoptosis by epigenetically inhibiting miR-34a. Sci Rep 5: 9787, 2015

3. Jemal A, Bray F, Center MM, Ferlay J, Ward E and Forman D Global cancer statistics. CA Cancer J Clin 61: 69-90, 2011.

4. Xie Z and Xu Z: General anesthetics and $\beta$-amyloid protein. Prog Neuropsychopharmacol Biol Psychiatry 47: 140-146, 2013.

5. Cakmakkaya OS, Kolodzie K, Apfel CC and Pace NL: Anaesthetic techniques for risk of malignant tumour recurrence. Cochrane Database Syst Rev 11: CD008877, 2014.

6. Almami A, Hegazy SA, Nabbi A, Alshalalfa M, Salman A Abou-Ouf H, Riabowol K and Bismar TA: ING3 is associated with increased cell invasion and lethal outcome in ERG-negative prostate cancer patients. Tumour Biol 37: 9731-9738, 2016.

7. He GH, Helbing CC, Wagner MJ, Sensen CW and Riabowol K Phylogenetic analysis of the ING family of PHD finger proteins. Mol Biol Evol 22: 104-116, 2005.

8. Wang Y, Dai DL, Martinka M and Li G: Prognostic significance of nuclear ING3 expression in human cutaneous melanoma. Clin Cancer Res 13: 4111-4116, 2007.

9. Doyon Y, Selleck W, Lane WS, Tan S and Côté J: Structural and functional conservation of the NuA4 histone acetyltransferase complex from yeast to humans. Mol Cell Biol 24: 1884-1896, 2004 .

10. Gunduz M, Ouchida M, Fukushima K, Ito S, Jitsumori Y, Nakashima T, Nagai N, Nishizaki K and Shimizu K: Allelic loss and reduced expression of the ING3, a candidate tumor suppressor gene at $7 \mathrm{q} 31$, in human head and neck cancers. Oncogene 21: 4462-4470, 2002.

11. Lu M, Chen F, Wang Q, Wang K, Pan Q and Zhang X: Downregulation of inhibitor of growth 3 is correlated with tumorigenesis and progression of hepatocellular carcinoma. Oncol Lett 4: 47-52, 2012

12. Gou WF, Sun HZ, Zhao S, Niu ZF, Mao XY, Takano Y and Zheng HC: Downregulated inhibitor of growth 3 (ING3) expression during colorectal carcinogenesis. Indian J Med Res 139: 561-567, 2014

13. Miyata T, Kodama T, Honma R, Nezu Y, Harada Y, Yogo T, Hara $\mathrm{Y}$ and Tagawa $\mathrm{M}$ : Influence of general anesthesia with isoflurane following propofol-induction on natural killer cell cytotoxic activities of peripheral blood lymphocytes in dogs. J Vet Med Sci 75: 917-921, 2013.

14. Song J, Shen Y, Zhang J and Lian Q: Mini profile of potential anticancer properties of propofol. PLoS One 9: e114440, 2014

15. Cui WY, Liu Y,Zhu YQ, Song T and Wang QS: Propofol induces endoplasmic reticulum (ER) stress and apoptosis in lung cancer cell H460. Tumour Biol 35: 5213-5217, 2014.

16. Su Z, Hou XK and Wen QP: Propofol induces apoptosis of epithelial ovarian cancer cells by upregulation of microRNA let-7i expression. Eur J Gynaecol Oncol 35: 688-691, 2014.
17. Huang H, Benzonana LL, Zhao H, Watts HR, Perry NJ, Bevan C, Brown R and Ma D: Prostate cancer cell malignancy via modulation of HIF-1 $\alpha$ pathway with isoflurane and propofol alone and in combination. Br J Cancer 111: 1338-1349, 2014.

18. Ye Z, Jingzhong L, Yangbo L, Lei C and Jiandong Y: Propofol inhibits proliferation and invasion of osteosarcoma cells by regulation of microRNA-143 expression. Oncol Res 21: 201-207, 2013.

19. Wang ZT, Gong HY, Zheng F, Liu DJ and Yue XQ: Propofol suppresses proliferation and invasion of gastric cancer cells via downregulation of microRNA-221 expression. Genet Mol Res 14: 8117-8124, 2015

20. Wang Y and Li G: ING3 promotes UV-induced apoptosis via Fas/caspase-8 pathway in melanoma cells. J Biol Chem 281: 11887-11893, 2006

21. Wang G, Zhang Q, Song Y, Wang X, Guo Q, Zhang J, Li J, Han Y, Miao Z and Li F: PAK1 regulates RUFY3-mediated gastric cancer cell migration and invasion. Cell Death Dis 6: e1682, 2015.

22. Heaney A and Buggy DJ: Can anaesthetic and analgesic techniques affect cancer recurrence or metastasis? Br J Anaesth 109 (Suppl 1): i17-i28, 2012.

23. Bajwa SJ, Anand S and Kaur G: Anesthesia and cancer recurrences: The current knowledge and evidence. J Cancer Res Ther 11: 528-534, 2015.

24. Vasileiou I, Xanthos T, Koudouna E, Perrea D, Klonaris C, Katsargyris A and Papadimitriou L: Propofol: A review of its non-anaesthetic effects. Eur J Pharmacol 605: 1-8, 2009.

25. Wang JW, Cheng WW, Xu T and Yang ZY: Propofol induces apoptosis and inhibits the proliferation of rat embryonic neural stem cells via gamma-aminobutyric acid type A receptor. Genet Mol Res 14: 14920-14928, 2015.

26. Xu YJ, Li SY, Cheng Q, Chen WK, Wang SL, Ren Y and Miao CH: Effects of anaesthesia on proliferation, invasion and apoptosis of LoVo colon cancer cells in vitro. Anaesthesia 71: 147-154, 2016.

27. Wang Y, Yang H, Hu Q, Hou Y, Luo H and Liu L: Effects of continuous sedation with propofol on peripheral blood mononuclear cell and intercellular adhesion molecule in beagles with combined burn-blast injuries. Zhonghua Yi Xue Za Zhi 94: 1573-1576, 2014 (In Chinese).

28. Zhang J, Zhang D, Wu GQ, Feng ZY and Zhu SM: Propofol inhibits the adhesion of hepatocellular carcinoma cells by upregulating microRNA-199a and downregulating MMP-9 expression. Hepatobiliary Pancreat Dis Int 12: 305-309, 2013.

29. Guo XG, Wang S, Xu YB and Zhuang J: Propofol suppresses invasion, angiogenesis and survival of EC-1 cells in vitro by regulation of S100A4 expression. Eur Rev Med Pharmacol Sci 19: 4858-4865, 2015.

30. Wang ZT, Gong HY, Zheng F, Liu DJ and Dong TL: Propofol suppresses proliferation and invasion of pancreatic cancer cells by upregulating microRNA-133a expression. Genet Mol Res 14: 7529-7537, 2015 .

31. Man YG, Zhou RG and Zhao B: Efficacy of rutin in inhibiting neuronal apoptosis and cognitive disturbances in sevoflurane or propofol exposed neonatal mice. Int J Clin Exp Med 8: 14397-14409, 2015.

32. Chen J, Chen W, Zhu M, Zhu Y, Xu P and Miao C: Angiotensin II-induced mouse hippocampal neuronal HT22 cell apoptosis was inhibited by propofol: Role of neuronal nitric oxide synthase and metallothinonein-3. Neuroscience 305: 117-127, 2015.

33. Chen G, Wang Y, Garate M,Zhou J and Li G: The tumor suppressor ING3 is degraded by $\mathrm{SCF}^{\mathrm{Skp2} 2-m e d i a t e d ~ u b i q u i t i n-p r o t e a s o m e}$ system. Oncogene 29: 1498-1508, 2010. 